EPJ Web of Conferences 71, 00137 (2014)

DOI: $10.1051 /$ epjconf / 20147100137

(C) Owned by the authors, published by EDP Sciences, 2014

\title{
Bringing high energy physics to the classroom with HY.P.A.T.I.A.
}

\author{
Stylianos Vourakis ${ }^{1}$ \\ ${ }^{1}$ Institute of Accelerating Systems and Applications' (IASA), P.O. Box 17214 \\ Greece
}

\begin{abstract}
School physics in most countries revolves around basic and rather old concepts that may be fundamental in understating physics but they are also very far from the cutting edge of modern research. HY.P.A.TI.A. with its various exercises aims to give students a taste of modern particle physics through the use of a specially designed interactive analysis tool in combination with real data from the ATLAS experiment at CERN.
\end{abstract}

\section{HY.P.A.T.I.A}

HY.P.A.T.I.A (HYbrid Pupil's Analysis Tool for Interactions in ATLAS) [1] is an event display that visualizes data from the ATLAS [2] experiment at CERN [3]. ATLAS is an experiment at the LHC [4], the world's largest and most powerful particle accelerator. ATLAS, along with the other experiments of the LHC is searching for answers to fundamental questions of physics such as the origin of mass, supersymmetry, extra dimensions etc. All this was built over the course of more than ten years by a collaboration consisting of scientists and engineers from more than 30 countries.

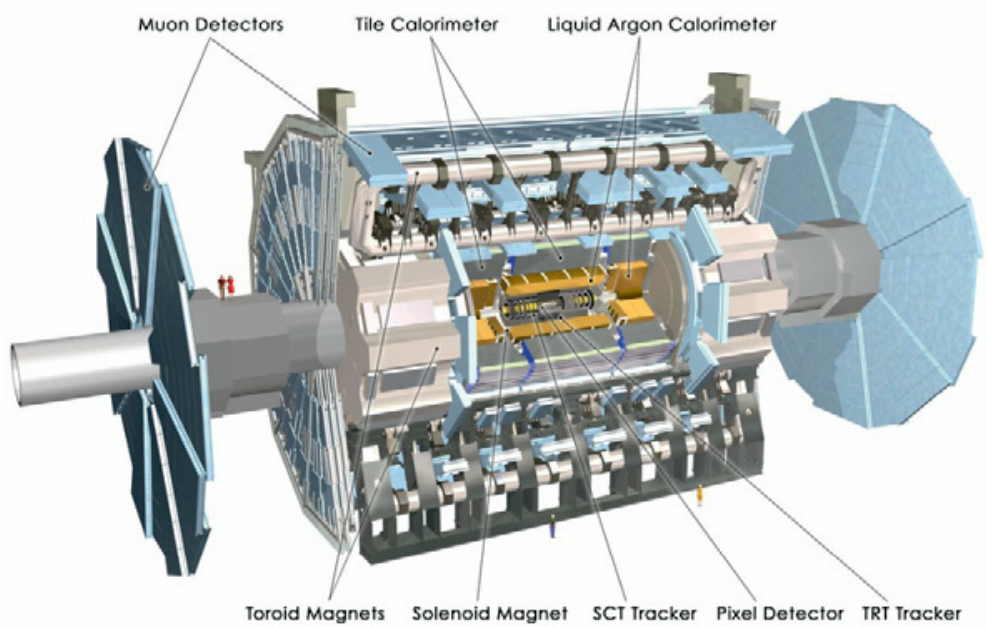

Figure 1. The ATLAS detector

This is an Open Access article distributed under the terms of the Creative Commons Attribution License 2.0, which permits unrestricted use, distribution, and reproduction in any medium, provided the original work is properly cited. 
ATLAS (Figure 1) is the largest of the LHC's experiments/detectors, with a length of more than $45 \mathrm{~m}$ and weight of about 7.000 tons. It has the size of a seven storey building and is perhaps the largest collaborative effort ever attempted in the physical sciences. Its members are 3,000 physicists from 177 institutes all over the world. ATLAS detects billions of collisions every day and a small fraction of those is recorded for later study. The registered products of the collisions are called "events" and are the data used by HYPATIA in various scientific and educational scenarios.

In general, high school students have very little knowledge about particle physics and modern physics in general as most of the school curriculum is focused on basic physics concepts which have been known for centuries. Nuclear and particle physics in particular, are very rarely mentioned in class. HYPATIA aims to demonstrate how a real researcher works and in the process give the students an interesting look at modern particle physics and cutting edge research and technology. It uses real events from the ATLAS experiment and follows the inquiry based teaching model.

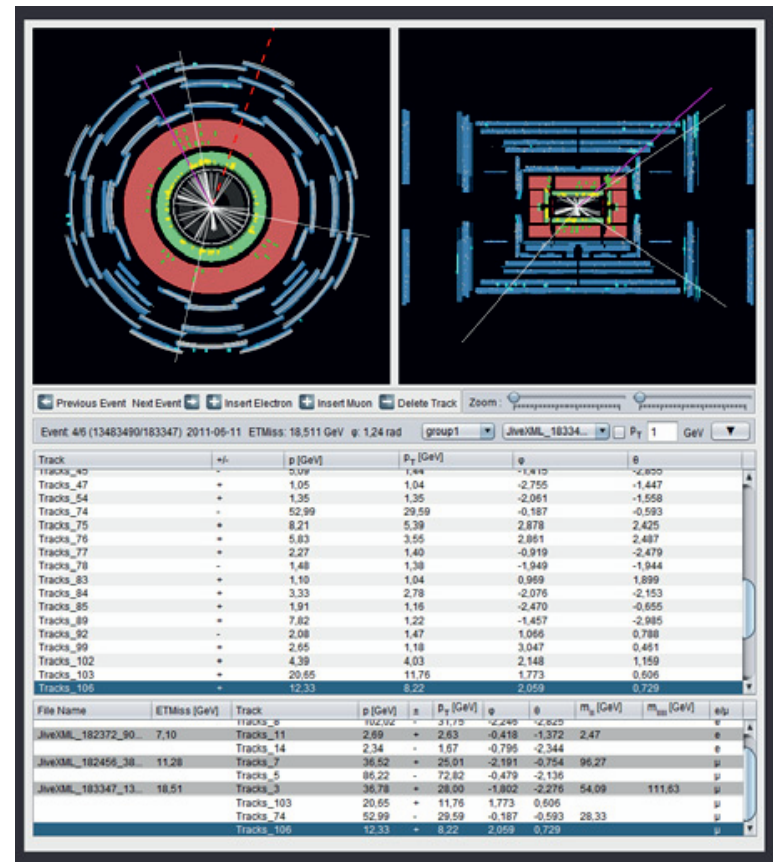

Figure 2. HY.P.A.T.I.A. applet

HYPATIA (Figure 2) offers a graphical representation of the products of proton collisions registered by the ATLAS detector. The students can interact/play with the events and in this way study the fundamental building blocks of nature and their interactions and at the same time learn how the gigantic state-of-the-art detectors work. This gives students a realistic and exciting look at the research being done at CERN and stimulates an enthusiastic interest in it and at possible scientific careers. The applet version of HYPATIA [1] is also very flexible in the sense that the educators can choose the level of difficulty they want to attain and at the same time adopt it for their own application according to the students' background. It has been tested through the "Discover the COSMOS" [5] EC-funded educational/coordination project in a large number of schools and by teachers from different countries. An advanced version of it is used at the University level and more specifically for the fourth year undergraduate laboratories by students of the Physics Department at the University of Athens [6] and also at the International Physics Masterclasses (Figure 3) (2008-2014) [7]. 


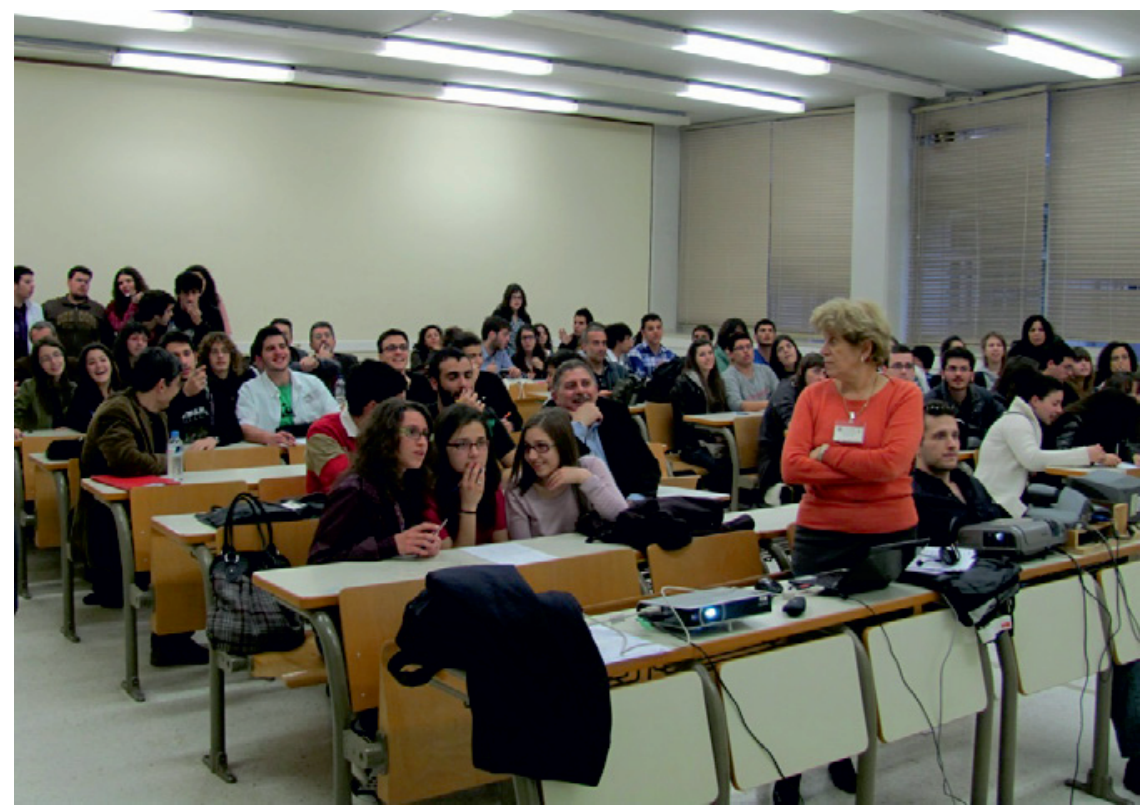

Figure 3. Students at the 2011 International Physics Masterclasses at the University of Athens

HYPATIA runs on almost any operating system and web browser as long as it supports java [8]. It requires very few resources and can be used freely from the University of Athens (UoA) website. The applet is quite simple and intuitive to use for the purpose of this exercise, but still basic computer skills are required. Figure 2 shows the overall view of the full version of the HYPATIA/applet. Our website is currently available in English, Greek and French with more languages coming soon. It contains all the information necessary (help, instructions, exercises etc.) for anyone to use the tools on their own. In particular, the instructional video tutorials for every exercise demonstrate the work that must be done by the students in order to complete the exercises.

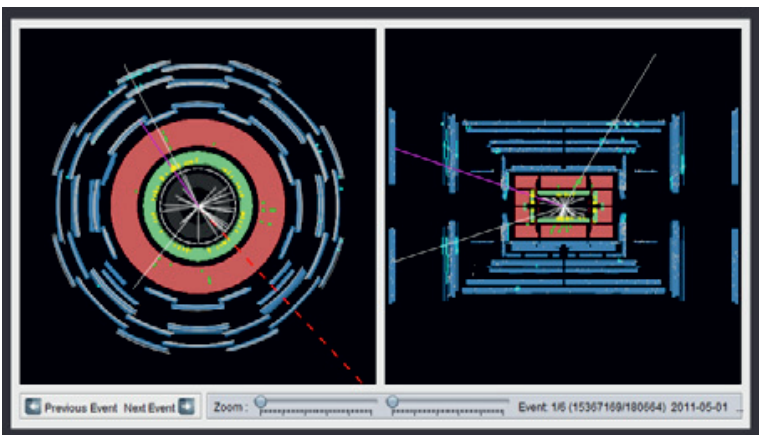

Figure 4. HY.P.A.T.I.A. applet, first level

The HYPATIA/applet supports four different versions aimed at various levels of users. The first level (Figure 4) consists mainly of the two canvas views which display cross sections of the ATLAS detector (one transverse to the LHC beams and one along the beams). The user can browse through different events and observe the number of tracks per event and their distribution in each view. This is a good starting point for entry level users, younger students, who only want to get an idea of what the ATLAS detector looks like, what do collisions of particles at the unprecedented energies produce and how their charged products ("tracks") are detected. 


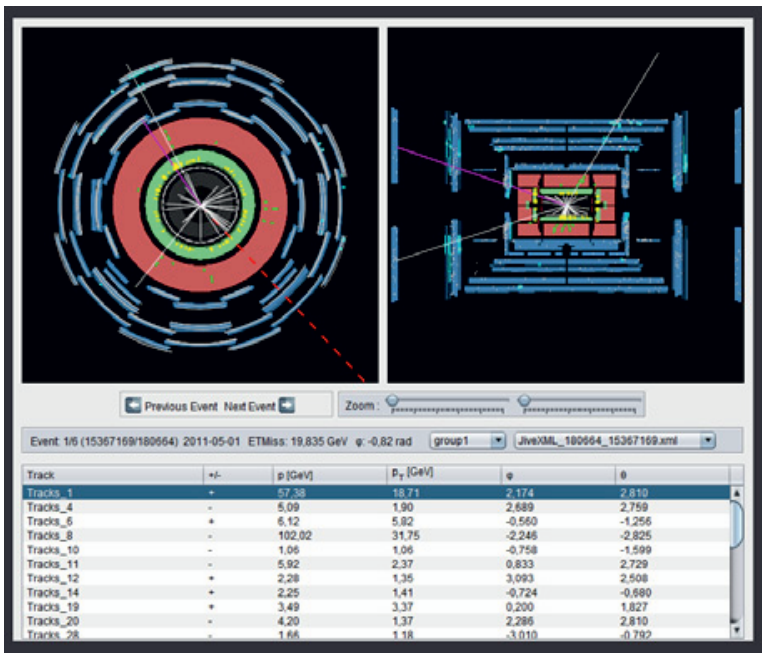

Figure 5. HY.P.A.T.I.A. applet, second level

The second level of the applet (Figure 5) adds the information about the track momenta and their directions in a tabular way. Here, the user is given instructions and examples and then is challenged to identify the different kinds of lepton tracks by using the signatures they leave in different parts of the detector. The muons should reach the outer detectors, the muon chambers (the blue chambers) in at least one of the views and the electrons should stop in the electromagnetic calorimeters (green ring) depositing considerable energy there (yellow marks) in at least one of the views. The user can study the properties (energy/momentum, direction and charge) of each track and also get indications about the momentum conservation in the transverse view by looking at a quantity called Etmiss.

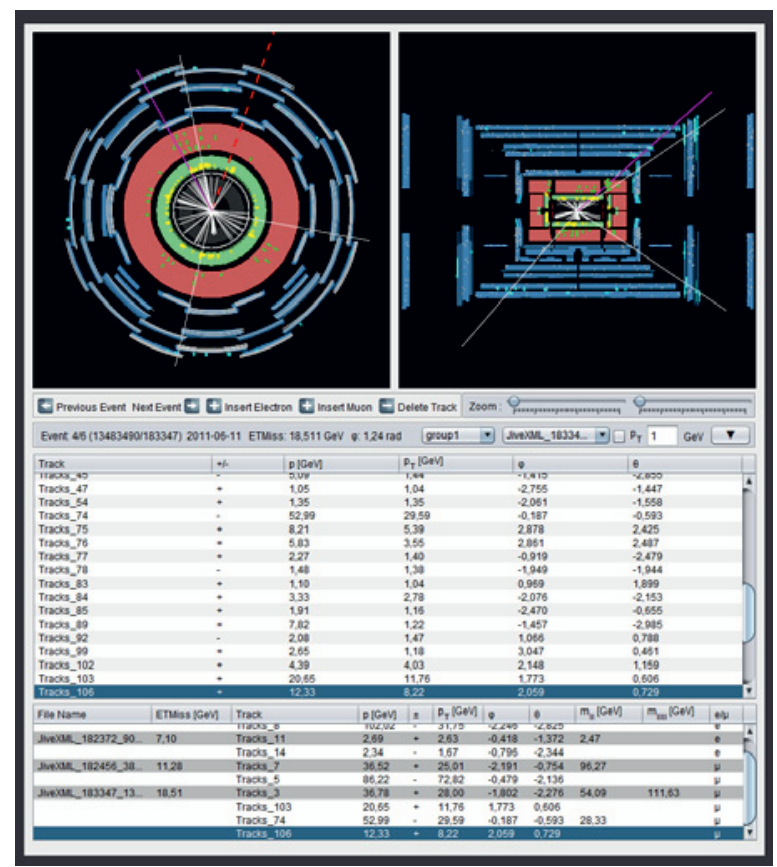

Figure 6. HY.P.A.T.I.A. applet, third level

The third level of the HYPATIA/applet (Figure 6) gives the user the possibility to combine tracks in order to search for invisible short-lived particles which decay into several tracks. In order to do that, 
the program calculates the invariant mass of the tracks chosen by the user and inserts its value in a corresponding table. When two or more particle tracks-traces in one "event", originate from the same point, which we call "vertex", they may belong to one original particle which decayed into these tracks. In order to check this hypothesis the user has to calculate the "invariant mass" of the original particle and investigate if these decay products came from the same particle. Examples of short lived particles are the carriers of the weak force, the $\mathrm{Z}^{0}$ and $\mathrm{W}$ bosons, as well as the newly discovered Higgs boson. An example of an easy to identify signature, is the decay of the $\mathrm{Z}^{0}$ boson to two muons or two electrons. If the pair of tracks originates from a $Z^{0}$ then their calculated invariant mass should be close to the mass of the $Z^{0}$, namely $91.2 \mathrm{GeV}$. This can be checked by plotting the relevant histogram of the invariant masses, a function which is available in the full version of the HYPATIA/applet.

For that purpose, the user has to find either an electron-positron or a muon-antimuon pair and use the "Insert electron" or "Insert muon" button twice to insert them into the invariant mass table. The resulting invariant mass value appears under column $\mathrm{m}_{1 \mathrm{l}}$. If the invariant mass corresponds to a twoelectron combination, an "e" is marked in the last column; otherwise if it corresponds to a two-muon combination, a " $\mu$ " is marked in the last column. Note that the decay to one muon and one electron combinations are not permitted because they violate the lepton number conservation. One can also delete tracks with the "Delete track" button and replace them with others.

The full version of the applet (Figure 2) gives access to the plotting of histograms of various kinematic variables which are generated automatically from the tracks that the user adds to the invariant mass table. This is the version which is used in the mini masterclasses.

\section{Activities}

HY.P.A.T.I.A. has been used by students and teachers in various activities since 2007 with great success and very positive feedback.

The mini masterclasses are events held at various schools and other institutes organized by the University of Athens/IASA, CERN and the Ellinogermaniki Agogi School. Their goal is to show the students how a real researcher works in the framework of a huge multinational collaboration of thousands of physicists. The format of the mini masterclasses is similar to that of the International Physics Masterclasses but of shorter duration. First the students are told about the basics of particle physics, the standard model and the work being done at CERN and ATLAS, by attending lectures given by the experts. Most of the times the students attend a "virtual visit" to the ATLAS experiment as well, where the scientists at the counting room of the experiment give the students a tour and answer questions posed by the students via videoconference. Following the virtual visit, the students (or a selected smaller group) are given instructions on how to analyze event data to look for $\mathrm{Z}^{0}$ and Higgs bosons using HYPATIA. The events that the students use in their analysis are real data from the ATLAS experiment. In the end, the students present their findings and compare them to those of their classmates and the expected results from the literature.

In this context, during the 2012-2013 school year, HY.P.A.T.I.A was used by more than 230 teachers and 600 students in 23 events all over Greece and also in a few selected events across Europe. The students and teachers got a chance to learn about particle physics and the fundamental building blocks of nature and also about the fundamentals of particle detector operation. They studied the way particles interact with the detectors and leave a characteristic signature according to their different types.

In addition to the mini masterclasses, HYPATIA was also used at the e-masterclasses initiatives. During these events students perform the HYPATIA analysis while guided remotely by the HYPATIA team from Athens and locally by their teachers. Two such pilot events took place last year with Dutch and Polish students visiting CERN. Two short videos [9], [10] which document the students' involvement and enthusiasm are available. 
The full version of HY.P.A.T.I.A. [11] has been part of the International Physics Masterclasses since 2008. In 2013 alone 70 institutes from 37 countries chose the Z-path and used HYPATIA in their activities with visiting school students and their teachers.

HY.P.A.T.I.A. along with the other analysis tools of particle physics, in the framework of the EC project Go-Lab [12], were presented as a hands-on experience at the 2013 CERN Open Days (Figure 7) [13] which were attended by more than 70.000 visitors.

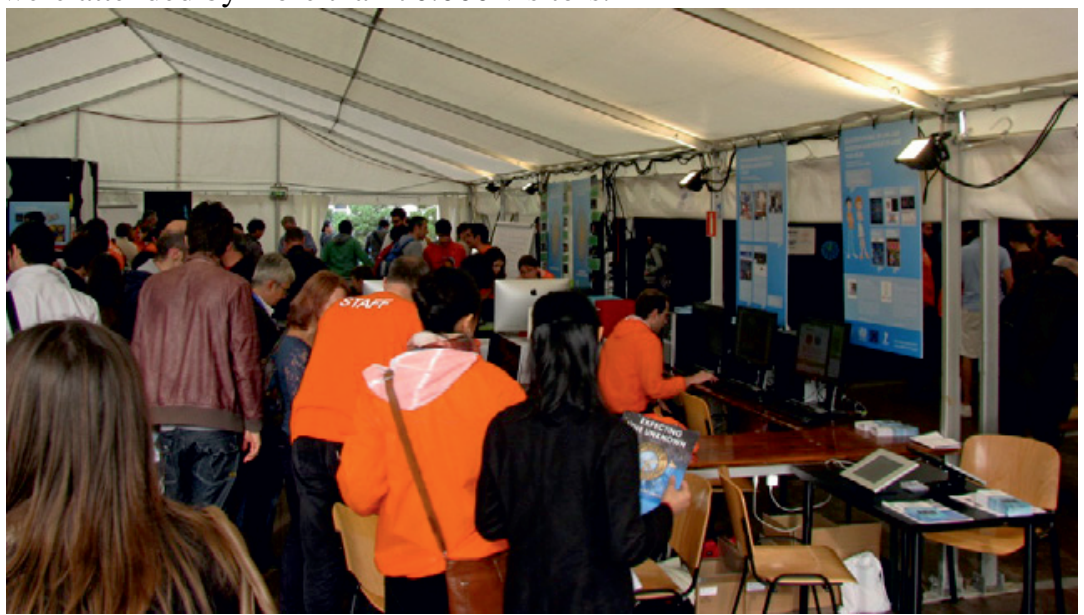

Figure 7. Visitors at the Go-Lab stand in the ATLAS tent during the 2013 CERN Open Days

Events like these, aim to demonstrate the process of research in order to make discoveries or experimentally measure basic quantities in nature (like the mass of the weak force carrier). They give the participants a realistic and exciting look at the research being done at CERN and stimulate an enthusiastic interest in it. They also aim to provide teachers with resources in order to inspire them to talk to their students about particle physics and show them a way to integrate it into their class at a level that is suitable to their students. They also show how scientific inquiry works in real life. Our long term aim is to drive the students to learn more on their own and investigate further and even pursue a career in physics.

\section{References}

1. [Online] http://hypatia.iasa.gr.

2. [Online] http://atlas.ch.

3. [Online] http://public.web.cern.ch/public/.

4. [Online] http://lhc-machine-outreach.web.cern.ch/lhc-machine-outreach/.

5. [Online] http://www.discoverthecosmos.eu.

6. [Online] http://www.phys.uoa.gr/.

7. [Online] http://www.physicsmasterclasses.org/.

8. [Online] http://www.java.com/.

9. [Online] http://home.web.cern.ch/students-educators/updates/2012/12/dutch-highschool-students-analyse-atlas-event-data.

10. [Online] http://home.web.cern.ch/students-educators/updates/2013/03/polishhigh-school-students-find-inspiration-cern.

11. [Online] http://hypatia.phys.uoa.gr/.

12. [Online] http://www.go-lab-project.eu/frontpage.

13. [Online] http://opendays2013.web.cern.ch/. 